\title{
Channel Estimation for Powerline MC-CDMA System
}

\author{
Thiyagu Kathiyaiah \\ Faculty of Engineering and Technology, Multimedia University Jalan Ayer Keroh Lama, Melaka, Malaysia \\ Email: thiyagu@mmu.edu.my
}

Received June 24, 2011; revised August 12, 2011 ; accepted June 14, 2012

\begin{abstract}
Successful development of broadband over powerline is obviously a potential solution for wired communication systems with the existence of the powerline network. From past research, it is known that the powerline channel suffers from multipath fading, frequency selectivity and also impulsive noise. Multi Carrier Code Division Multiple Access (MC-CDMA) is a promising solution for an impulsive noise powerline channel. This paper starts with the MC-CDMA transmitter structure and focuses on powerline channel model, noise model and various types of available channel estimators. The main concern in Powerline Communication Systems is the existence of impulsive noise. The proposed pilot assisted channel estimation uses the modified least square estimator that reduces the effect of impulsive noise in the estimated channel impulse response.
\end{abstract}

Keywords: Channel Estimation; MC-CDMA; PLC; LS

\section{Introduction}

Power line communication or power line carrier (PLC) is a system for carrying data on a conductor also used for electrical power transmission. Broadband over power lines (BPL) uses PLC by sending and receiving information bearing signals over power lines to provide access to the Internet [1].

The major advantage offered by power line based home networks is the availability of an existing infrastructure of wires and wall outlets so that frequent revision or new cable installation is averted [2].

The power line network differs considerably in topology, structure, and physical properties from conventional media such as twisted pair, coaxial, and fiber-optic cables. Considerable effort has been devoted recently to characterize the PLC channel. However, ascertaining the transfer function is not an easy task since power line characteristics may change due to the particular topology of a given link and adjoining circuits. Several approaches have been followed for characterizing the PLC channel [3-5]. In particular, an approach that considers the multipath effects is given in [4]. The multipath nature of the power line channel arises from the presence of several branches and impedance mismatches that cause multiple reflections.

There are some factors that contribute to the distortion and noise in the received signals in power line networks. These factors are mainly the topology of the main network, multipath signal propagation, cable losses as well as the colored noise [4]. In the indoor network, numerous reflections are caused by the joints of the house service cables, house connection boxes, and the joints at series connections of cables with different characteristic impedance [4].

Multi carrier code division multiple access (MCCDMA) system is well known for its effectiveness in combating multipath and intersymbol interference (ISI) effects. It can accommodate very high data rate transmission over hostile channel. In MC-CDMA system, a data symbol is transmitted over multiple narrowband subcarriers to achieve frequency diversity [6]. The basic principle of this technique is that frequencies separated by more than the coherence bandwidth of the channel will be uncorrelated and will thus not undergo the same fades. In order to achieve high spectral efficiency, the spacing between the subcarriers is kept as close as possible while their orthogonality is still maintained by adding a cyclic prefix to the transmitted vector. The length of the cyclic prefix has to be as large as the length of the channel impulse response.

Channel estimation in up-link MC-CDMA systems is mathematically equivalent to that in multiple input multiple output (MIMO) orthogonal frequency division multiplexing (OFDM) systems [7,8]. Channel estimation for MIMO_OFDM has been investigated in [9], which can be used for up-link MC-CDMA systems. Channel estimation for up-link MC-CDMA systems with prefiltering has been investigated in $[7,8]$.

The aim of this paper is to design an improved channel estimator for power line communication systems to support next-generation multimedia services. The conven- 
tional channel estimation algorithms are designed on the assumption of Gaussian channel models. The proposed least square estimator removes the impulsive noise component of the estimated channel to provide better bit error rate performance. The results are discussed in the Results section.

\section{Powerline Communication System}

\subsection{Channel Model}

The power line communication channel exhibits multipath propagation phenomenon. This is due to multiple lines with different characteristic impedances that are connected to the electrical network. Therefore, in this paper, the PLC channel is modeled as the multipath channel proposed in [4]. Power line channel exhibits different types of noises including narrowband noise, colored noise, white Gaussian noise and also impulsive noise. According to [4], the frequency domain channel transfer function can be described as

$$
H(f)=\sum_{i=1}^{N} g_{i}\left(e^{-\left(a_{0}+a_{1} f^{k}\right) d_{i}}\right)\left(e^{-\left(j 2 \pi f\left(d_{i} / v_{P}\right)\right.}\right)
$$

where $g_{i}$ is the weighting factor for the $i^{\text {th }}$ path, $\left\{a_{0}, a_{1}\right\}$ are attenuation factors, $k$ is the exponent of the attenuation factor, $N$ is the number of paths, $d_{i}$ is the length of the $i^{\text {th }}$ path, and $v_{p}$ is the propagation velocity of the cable.

Figures 1(a) and (b) show the impulse responses for 4-path and 15-path PLC channels respectively. Higher number of multiple paths contributes to higher signal distortion.

\subsection{Noise Model}

The indoor power line channel is a time-varying frequency selective fading channel with interference due to the colored and impulsive noise generated by electrical appliances and external sources. Multipath response due to the power cable layout and loading conditions is also a problem. Nevertheless, the worst signal impairment is due to the short duration and high peak impulsive noise. To model the noise in PLC, we use a Gaussian mixture model .The probability density function (PDF) of the impulsive noise can be described as

$$
f=(1-\varepsilon) N\left(0, \sigma^{2}\right)+\varepsilon N\left(0, k \sigma^{2}\right)
$$

where $\varepsilon$ represents the probability of impulse occurrence, $N\left(0, \sigma^{2}\right)$ is the Gaussian noise component with zero mean and variance $\sigma^{2}$, and $N\left(0, k \sigma^{2}\right)$ is the impulsive noise component with zero mean and variance $k \sigma^{2}$. The noise signal becomes highly impulsive with increasing in $\varepsilon$, the probability of impulsive occurrence. The plot of impulsive noise for different values of

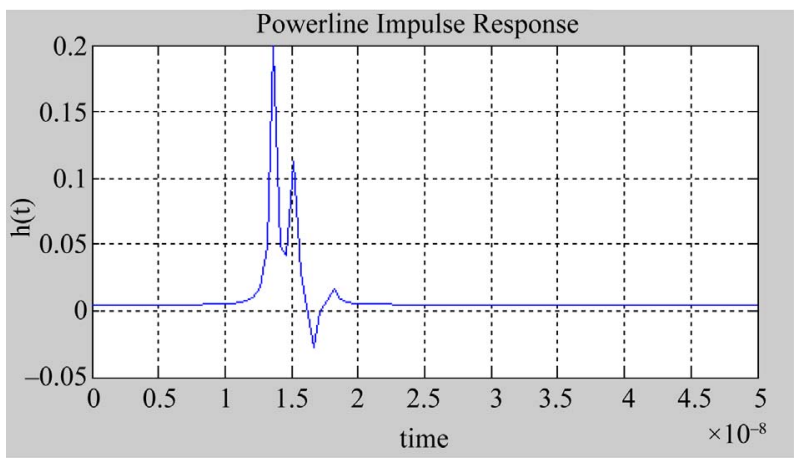

(a)

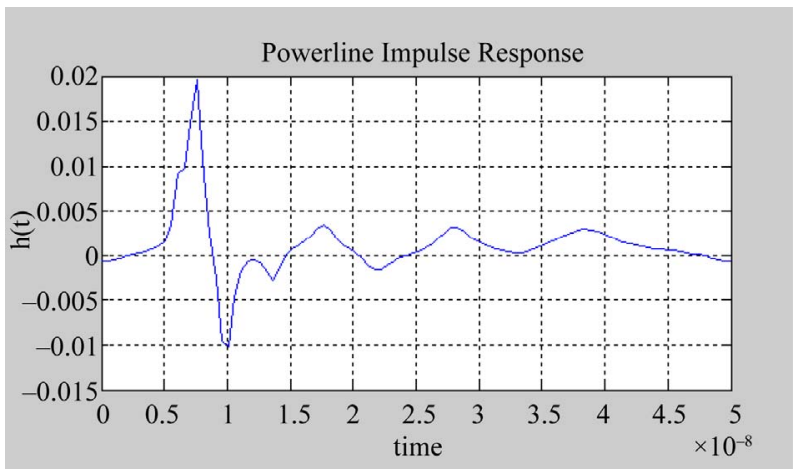

(b)

Figure 1. (a) Impulse response for 4-path PLC channel; (b) Impulse response for 15-path PLC channel.

$\varepsilon$ is depicted in Figure 2. It can be clearly seen that the signal becomes highly impulsive when $\varepsilon$ is equals to 1 . Therefore a careful study is needed to reduce the impact of impulsive noise on the transmitted signal.

\subsection{PLC Transmission System}

The MC-CDMA system employs $N$ subcarriers and each user transmit $M$ bits during a signaling interval, leading to the spreading factor or processing gain, $G$, of $N / M$. Figure 3 shows the structure of the transmitter. The user data stream, $u$ is converted into $M$ parallel streams and each stream is spread with the aid of orthogonal spreading sequences (e.g., Walsh Hadamard codes) which maps the same bit to $G$ subcarriers. The spacing between the subcarriers conveying the same bit is set to $M$ to reduce the correlation of the fading of these subcarriers. For simplicity, only single user is depicted in Figure 4, but a maximum of $G$ users can be incorporated. The $u^{\text {th }}$ user's spreading sequence, $c_{g}^{u}, g=0,1,2 \cdots G-1$, is orthogonal to other users' spreading code. In this model, each user transmits its data at the rate of $M / T$ bps, where $T$ is the frame duration. If the transmission rate can be lowered, the maximum number of users, $U$, can be increased such that the data generated by the $M$ users replace the 1 : $M$ serial-to-parallel converter. In this case, the total number of users supported can reach the total number of subcarriers, yielding $U=N$. 

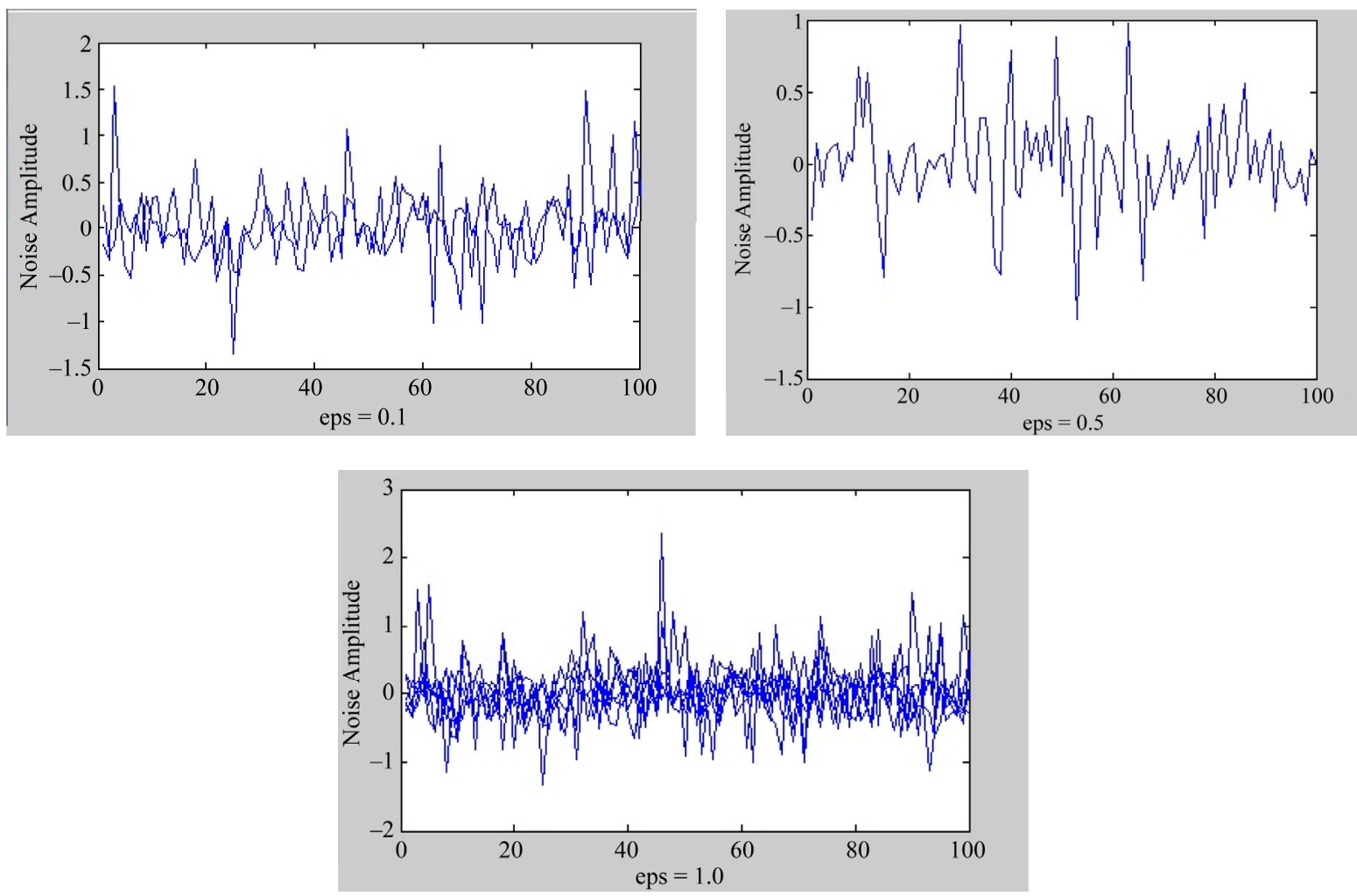

Figure 2. Noise amplitude plot for Gaussian mixture model for $\varepsilon=0.1,0.5$, and 1.0.

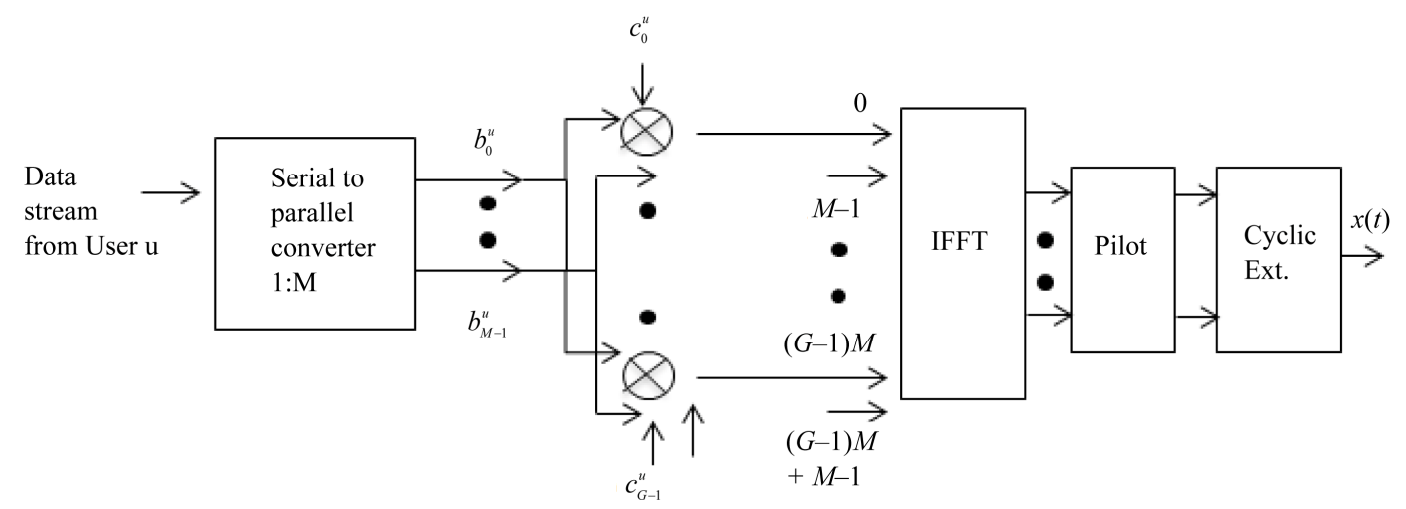

Figure 3. MC-CDMA transmitter structure.

The complex baseband representation of the transmitted signal, $X(t)$ can be expressed as:

$$
X(t)=\sum_{u=0}^{U-1} \sum_{m=0}^{M-1} \sum_{g=0}^{G-1} \sqrt{E_{c}} b_{m}^{u} c_{g}^{u} e^{j 2 \pi \frac{1}{T}(g M+m) t}
$$

where

$U$ is the number of users, which has a maximum of $G$;

$M$ is the number of bits transmitted per user;

$G$ is the spreading factor or the processing gain given as $N / M$, where $N$ is the number of subcarriers;

$E_{c}$ is the energy per subcarrier, or chip, and $E_{c}=E_{b} / N$, where $E_{b}$ is the energy per bit before spreading;

$T$ is the signaling interval, during which $M$ numbers of bits per user are transmitted and $1 / T$ is equal to the spacing between adjacent subcarriers;

$b_{m}^{u} \in\{ \pm 1\}$ is the $m^{\text {th }}$ bit of user $u$;

$c_{g}^{u} \in\{ \pm 1\}$ is the $g^{\text {th }}$ chip of the $u^{\text {th }}$ user's spreading sequence.

Pilot symbols and cyclic prefix are then added to the baseband signal. This signal is then transmitted through the PLC channel. The demodulation at the receiver is done after the removal of the cyclic prefix. The received vector can be represented by

$$
\begin{gathered}
Y=X h+n \\
Y=\left[\begin{array}{llll}
y(a) & y(a+1) & \cdots & y(L)
\end{array}\right]^{T}
\end{gathered}
$$




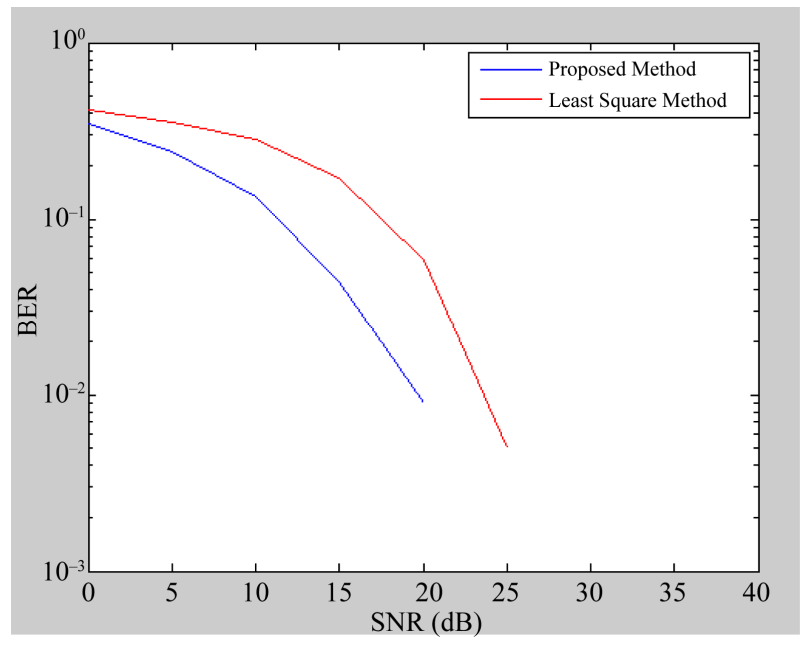

Figure 4. Performance evaluation of conventional least square and proposed method.

$$
\begin{gathered}
X=\left[\begin{array}{cccc}
x(a) & x(a-1) & \cdots & x(1) \\
x(a+1) & x(a) & \cdots & x(2) \\
\vdots & \vdots & \vdots & \vdots \\
x(L) & x(L-1) & \cdots & x(L-a+1)
\end{array}\right] \\
h=\left[\begin{array}{llll}
h_{1} & h_{2} & \cdots & h_{a}
\end{array}\right]^{T} \\
n=\left[\begin{array}{llll}
n(a) & n(a+1) & \cdots & n(L)
\end{array}\right]^{T}
\end{gathered}
$$

where

$X$ is the transmitted signal;

$h$ is the powerline channel impulse response;

$n$ is the Gaussian mixture noise;

$L$ is the received signal length;

$a$ is the channel impulse response length.

\section{Channel Estimation}

\section{Least Square Method}

The estimation of the impulse response of the PLC channel is carried out by transmitting some pilot symbols as shown in Figure 5. Pilot symbol-assisted schemes obtain a transfer function estimate on the basis of known time domain pilot symbols that are interspersed with the transmitted data symbols. For each received pilot subcarrier the corresponding channel transfer function value is estimated as the quotient of the received and the expected subcarrier value.

The received signal vector can be written as

$$
Y_{P}=X_{p} h+n
$$

where $X_{p}$ denotes the transmitted pilot symbols, $h$ and $n$ denote the channel impulse response and gaussian mixture noise respectively. The conventional least square estimate of the unknown channel is

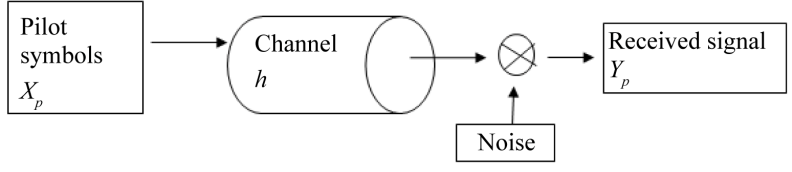

Figure 5. Channel estimation with pilot symbol insertion.

$$
\tilde{h}=\left(X_{p}^{H} X_{p}\right)^{-1} X_{p}^{H} Y_{p}
$$

If $X_{p} \geq a$, the lease square estimate of the channel impulse response is given by (Substitute $Y_{p}=X_{p} \times h+n$ into (10))

$$
\begin{aligned}
& \tilde{h}=\left(X_{p}^{H} X_{p}\right)^{-1} X_{p}^{H}\left(\left(X_{p} h\right)+n\right) \\
& \left.\tilde{h}=\left(X_{p}^{H} X_{p}\right)^{-1} X_{p}^{H}\left(X_{p} h\right)+\left(X_{p}^{H} X_{p}\right)^{-1} X_{p}^{H} n\right) \\
& \tilde{h}=h+\left(X_{p}^{-1} n\right)
\end{aligned}
$$

From Equation (11) the additional $X_{p}^{-1} n$ term is actually the frequency response of the noise component and may be expressed as

$$
v=X_{p}^{-1} n
$$

Vector $v$ is a sufficient statistics to calculate the frequency response of the impulse noise on pilot subcarriers, and thus its impact to the channel estimate. If $X_{p}$ is larger than the noise impulsive components, by definition $v$ can preserve the sparse property of $f$, that is, only few positions will have large values dominated by the impulsive components, while other positions will have smaller value corresponding to Gaussian noise components.

Now we can rewrite (11) as

$$
\tilde{h}=h+v
$$

It is clear that the perfect channel impulse response is obstructed by the second term $v$. The main objective is to estimate the unknown $v$, and mitigate the impact of the impulse noise on the channel impulse response by cancelling the second part, $v$.

The unknown $v$ can be estimated by first calculating the difference between the received signals at $X_{p}$ pilot subcarriers and the recovered signal form $\tilde{h}$. The difference $\hat{n}$ can be expressed as

$$
\hat{n}=Y_{p}-X_{p}(\tilde{h})
$$

The value of $\hat{n}$ is depends on interference. The Gaussian noise component in $\hat{n}$ are very sensitive to interference from impulsive noise components, since the interference from impulsive noise component can supersede the Gaussian noise component due to the large power difference between them.

We will have to prune $(\dot{n})$ out the values of $\hat{n}$ dominated by interference at background noise position prior to cancellation. We can use the simple clipping method where the threshold is set to three times the median of $\hat{n}$ 
which can effectively tear apart impulsive noise component and Gaussian noise component.

Finally we cancel the impact of the estimated impulse noise component on $\tilde{h}$ to get the new channel estimate:

$$
\overline{\bar{h}}=\tilde{h}-\left(X_{p}^{-1}\right) \dot{n}
$$

where, $\overline{\bar{h}}$ is the estimated channel impulse response obtained with the proposed method.

Based on Equation (15), $\overline{\bar{h}}$ is the newly estimated channel impulse response with minimum impact from impulsive noise component.

\section{Results}

The simulation is carried out by transmitting 100 symbols in 64 subcarriers. The size of pilot symbol is 100 bits with a cyclic prefix of $25 \%$. The obtained BER curve is plotted in Figure 4. The is significant improvement in the BER curve plot. The proposed method gives improvement about $6 \mathrm{~dB}$ to the BER curve.

\section{Conclusion}

In this paper, an MC-CDMA transmission system with proposed channel estimation at the receiver is more robust against the impulsive noise existing in the power line channel.

\section{REFERENCES}

[1] S. Galli, A. Scaglione and K. Dostert, "Broadband Is Power: Internet Access through the Power Line Network," IEEE Communications Magazine, Vol. 41, No. 5, 2003, pp. 82-83. doi:10.1109/MCOM.2003.1200105
[2] S. Galli, K. Kerpez, S. Ungar and D. Waring, "Home Networks and Internet Appliances Shape Service Provider Access Architectures," Proceeding of the International Symposium on Services and Local Access, Stockholm, 18-23 June 2000.

[3] H. Philipps, "Modeling of Power Line Communications Channels," Proceeding of IEEE International Symposium on Power Line Communications and Its Applications, Lancaster, April 1999.

[4] M. Zimmermann and K. Dostert, “A Multipath Model for the Power Line Channel," IEEE Transactions on Communications, Vol. 50, No. 4, 2002, pp. 553-559. doi:10.1109/26.996069

[5] H. Philipps, "Development of a Statistical Model for Power Line Communications Channels," Proceeding of IEEE International Symposium on Power Line Communications and Its Applications, Limerick, April 2000.

[6] M. Zimmermann and K. Dostert, "Analysis and Modeling of Impulsive Noise in Broad-Band Powerline Communications," IEEE Transactions on Electromagnetic Compatibility, Vol. 44, No. 1, 2002, pp. 249-258. doi:10.1109/15.990732

[7] L. Sanguinetti and M. Morelli, "Channel Acquisition and Tracking for MC-CDMA Uplink Transmission," IEEE Transactions on Vehicular Technology, Vol. 55, No. 3, 2006, pp. 956-967. doi:10.1109/TVT.2005.863354

[8] L. Sanguinetti, I. Cosovic and M. Morelli, "Channel Estimation for MC-CDMA Uplink Transmissions with Combined Equalization," IEEE Journal on Selected Areas in Communications, Vol. 24, No. 6, 2006, pp. 1167-1178. doi:10.1109/JSAC.2005.864023

[9] Y. Li, N. Seshadri and S. Ariyavisitakul, "Channel Estimation for OFDM Systems with Transmitter Diversity in Mobile Wireless Channels," IEEE Journal on Selected Areas in Communications, Vol. 17, No. 3, 1999, pp. 461471. 